\title{
Internal Energy and Parameters of the Order-Disorder Phase Transition in Titanium Monoxide $\mathrm{TiO}_{y}$
}

\author{
M. G. Kostenko ${ }^{a}$, A. A. Rempel ${ }^{a, b}$, and A. V. Lukoyanov ${ }^{b, c}$ \\ ${ }^{a}$ Institute of Solid-State Chemistry, Ural Branch, Russian Academy of Sciences, Yekaterinburg, 620090 Russia \\ ${ }^{b}$ Ural Federal University, Yekaterinburg, 620002 Russia \\ ${ }^{c}$ Institute of Metal Physics, Ural Branch, Russian Academy of Sciences, Yekaterinburg, 620990 Russia \\ e-mail: rempel@ihim.uran.ru \\ Received December 28, 2012
}

\begin{abstract}
Quantum-mechanical ab initio calculations are used to simulate the free energy functions for titanium monoxide $\mathrm{TiO}_{y}$. The effect of the long-range order of the $\mathrm{Ti}_{5} \mathrm{O}_{5}$ type superstructure on the internal energy of the compound is studied by the supercell method. The dependences of the configuration entropy and free energy on the long-range order parameter are determined. It is found that the order-disorder phase transition in titanium monoxide must occur in accordance with the mechanism of the first-order phase transition with a critical value of the long-range order parameter of 0.971 . The calculated parameters of the phase transition are compared with the experimental data and the results obtained using the model of point charges and by calculating the Madelung energy. It is concluded that the short-range order and the phonon entropy must be taken into account in calculating the equilibrium phase diagrams for strongly nonstoichiometric compounds.
\end{abstract}

DOI: $10.1134 / \mathrm{S} 1063776113060071$

\section{INTRODUCTION}

Many binary nonstoichiometric nitrides, carbides, and oxides of transition metals are interesting not only because of the high concentration of structural vacancies, but also the possibility of their ordering [1]. The ordering effect on the properties of a compound can be comparable with the nonstoichiometry effect. One of the problems encountered in strongly nonstoichiometric compounds [1] is allowance for their ordering in calculating the equilibrium concentration-temperature phase diagrams.

To calculate the equilibrium phase diagram, we must solve the system of two equations for each value of temperature $T$ [1],

$$
\begin{gathered}
\frac{\partial F_{\mathrm{dis}}\left(y_{1}, T\right)}{\partial y_{1}}=\frac{\partial F_{\text {ord }}\left(y_{2}, T\right)}{\partial y_{2}}, \\
F_{\text {dis }}\left(y_{1}, T\right)-y_{1} \frac{\partial F_{\mathrm{dis}}\left(y_{1}, T\right)}{\partial y_{1}} \\
=F_{\text {ord }}\left(y_{2}, T\right)-y_{2} \frac{\partial F_{\text {ord }}\left(y_{2}, T\right)}{\partial y_{2}},
\end{gathered}
$$

where $F_{\text {dis }}$ and $F_{\text {ord }}$ are the free energies of the disordered and ordered phases, respectively, and $y_{1}$ and $y_{2}$ are the contents of the disordered and ordered phases of the $\mathrm{MX}_{y}$ binary compound. The free energy is known to be defined as

$$
F=E-T S,
$$

where $E$ is the internal energy of the system and $S$ is the entropy. At a given temperature $T$, the phase with the minimal free energy is the equilibrium phase. If, however, both phases are in equilibrium, the amount and composition of each phase are determined by the solutions to Eqs. (1) and (2).

The degree of ordering of structural vacancies is characterized by long-range order parameters $\eta^{M}$ and $\eta^{X}$ of the metallic and nonmetallic sublattices [1]. If the compound is completely ordered, the long-range order parameters are equal to unity. In the case of the completely disordered state, the long-range order parameters are equal to zero. Each value of the longrange order parameters corresponds to a certain free energy of the compound:

$$
F\left(\eta^{M}, \eta^{X}\right)=E\left(\eta^{M}, \eta^{X}\right)-T S\left(\eta^{M}, \eta^{X}\right) .
$$

Thus, the problem is reduced to determining the analytic dependences of type (4) for each composition $y$.

In the cluster variation method proposed by Kikuchi [2-6], the free energy of the compound being ordered and consisting of $N$ atoms is expressed as

$$
F=N \sum_{s=1}^{n} o^{(S)} \sum_{i \in S} \lambda_{i}^{(S)} P_{i}^{(S)}\left[\varepsilon_{i}^{(S)}+k_{\mathrm{B}} T \ln P_{i}^{(S)}\right],
$$

where $P_{i}^{(S)}$ are the probabilities of the $i$ th configurations of a basis cluster $S$; $(n-1)$ is the number of overlap figures; $o^{(S)}$ and $\lambda_{i}^{(S)}$ are the revaluation and multiplicity parameters, respectively; and $\varepsilon_{i}$ are the energies 
of the $i$ th configurations. From all quantities appearing in expression (5), only probabilities $P_{i}^{(S)}$ depend on the long-range order parameters. The method for calculating the probabilities of configurations of basis clusters as functions of the long-range order parameters was described in [7-9]. The corresponding analytic expressions for octahedral clusters in the $B 1$ basis structure were derived in [9] for ordering of the $M_{5} X_{5}$ type.

A significant problem to be solved in the cluster variation method is associated with the choice of energy parameters $\varepsilon_{i}$ for each configuration of the basis cluster. A solution to this problem was proposed in the order parameter functional method [6, 10-12], which was used for the first time to calculate the phase diagrams for many nonstoichiometric compounds taking into account the atomic-vacancy ordering [13-16]. The disadvantages of the cluster variation method, as well as the order parameter functional method, are the impossibility of taking into account the long-range interaction between atoms of the crystal and considerable computational difficulties in the case when ordering takes place not in one but in two sublattices simultaneously.

Another method involves simulation of the free energy function using quantum-mechanical ab initio calculations. To take into account the disorder in the arrangement of atoms and vacancies in band structure calculations, the coherent potential approach (CPA) or a superlattice method should be used. The coherent potential approach can be used to analyze the electronic structure of disordered nonstoichiometric compounds with any concentration of vacancies; however, this method does not permit taking into account static displacements of atoms and determining the correct values of the internal energy of the system. On the other hand, the supercell method cannot be used to simulate a completely disordered structure of nonstoichiometric compounds with an arbitrary concentration of vacancies in the sublattices. The accuracy in simulating disorder and the number of compositions accessible for analysis depend on the superlattice size, which is limited by the capabilities of computers. Nevertheless, the supercell method can be successfully used for compounds with a high concentration of vacancies. The effect of nonstoichiometry on the electronic structure of titanium monoxide $\mathrm{TiO}_{y}$ containing from $1 / 6$ to $1 / 4$ fractions of vacancies in the sublattices was investigated in [17] with the supercell method. The supercell chosen for analysis was four times as large as the unit cell of the ordered titanium monoxide $\mathrm{Ti}_{5} \mathrm{O}_{5}$. The calculated value of the enthalpy of formation of the disordered state was in agreement with the experimental data [18].

$\mathrm{Ab}$ initio calculations had not been used earlier for analyzing the dependence of the internal energy on the long-range order parameter for strongly nonstoichiometric compounds. Thermodynamic studies were limited to comparing the energies of completely disordered and completely ordered states, for example, in [19]. The ordered and disordered structures were studied by different methods, which rendered the comparison of their energies incorrect.

In [20], the point charge model was used for calculating the energy parameters of nonstoichiometric titanium oxide $\mathrm{TiO}_{y}$; it was shown that the Madelung energy is a quadratic function of the long-range order parameter. However, the calculated value of the phasetransition temperature is an order of magnitude higher than the experimental value. The decrease in the Madelung energy of the crystal can be considered as a reason for the order-disorder phase transition; however, to calculate the phase transition parameters correctly, detailed features of the interaction between atoms following from the Schrödinger equation should be taken into account.

In this connection, this study aims at determining the dependence of the internal energy on the longrange order parameter proceeding from quantummechanical $\mathrm{ab}$ initio calculations and at calculating the order-disorder phase transition parameters for titanium monoxide on the basis of this dependence. From all strongly nonstoichiometric compounds, titanium monoxide is the most suitable object for such an analysis, because it is characterized by a high concentration of vacancies in the sublattices (exceeding 15\% [21-23]) and a wide homogeneity range (from $y=$ 0.70 to 1.25 [22]). In addition, in contrast, for example, to niobium monoxide, vacancies in $\mathrm{TiO}_{y}$ can be both ordered and disordered.

\section{CALCULATION OF INTERNAL ENERGY}

Titanium monoxide has the basic structure of the $B 1$ type. In the disordered cubic phase, which is in thermodynamic equilibrium at temperatures above $1263 \mathrm{~K}[21,22]$, vacancies are distributed randomly over the sites of the basis structure. Upon slow annealing below $1263 \mathrm{~K}$, vacancies are redistributed over the sites of the sublattices of the basis structure. As a result of such a redistribution, various ordered phases are formed [21-24]. If $y=1.0$, the $\mathrm{Ti}_{5} \mathrm{O}_{5}$ monoclinic superstructure is formed as a result of ordering. Its unit cell is shown in Fig. 1. Each sublattice of this superstructure contains $1 / 6$ of vacant sites.

In the disordered phase, only two sublattices (titanium and oxygen) can be singled out, while in the ordered phase of $\mathrm{Ti}_{5} \mathrm{O}_{5}$, four sublattices (titanium atoms, titanium vacancies, oxygen atoms, and oxygen vacancies) should be singled out. The degree of ordering of the compound is determined by the distribution functions for the titanium and oxygen sublattices of the basic structure:

$$
\begin{aligned}
& n_{\mathrm{Ti}}=f\left(x_{I}, y_{I}, z_{I}, \eta^{\mathrm{Ti}}\right), \\
& n_{\mathrm{O}}=f\left(x_{I}, y_{I}, z_{I}, \eta^{\mathrm{O}}\right) .
\end{aligned}
$$


Here, $n_{\mathrm{Ti}}$ and $n_{\mathrm{O}}$ are the probabilities of finding a titanium or an oxygen atom, respectively, at the site of the $B 1$ basis structure with coordinates $\left(x_{I}, y_{I}, z_{I}\right) ; \eta^{\mathrm{Ti}}$ and $\eta^{\mathrm{O}}$ are the long-range order parameters for the titanium and oxygen sublattices, respectively. In the completely ordered structure, $n_{\mathrm{Ti}}=1$ for the sublattice of titanium atoms and $n_{\mathrm{Ti}}=0$ for the sublattice of titanium vacancies. Analogously, $n_{\mathrm{O}}=1$ for the sublattice of oxygen atoms and $n_{\mathrm{O}}=0$ for the sublattice of oxygen vacancies. The form of functions (6) and (7) for the four sublattices of the $\mathrm{Ti}_{5} \mathrm{O}_{5}$ superstructure is given in Table 1.

The long-range order parameters can possess the values from zero to $\eta_{\max }$, where $\eta_{\max } \leq 1$. The values of $\eta_{\max }$ are determined by normalization of functions (6) and (7) to the composition of the compound; i.e., for the given composition $y$, the values of the distribution function should not be smaller than zero or greater than unity. Here, we will consider the equiatomic composition $(y=1)$, for which the long-range order parameter can assume values from 0 to 1 . It follows from symmetry considerations as well as from the results of experiments [23] that the long-range order parameters for the titanium and oxygen sublattices in the equilibrium ordered phase of $\mathrm{Ti}_{5} \mathrm{O}_{5}$ are identical $\left(\eta^{\mathrm{Ti}}=\eta^{\mathrm{O}}=\eta\right)$. Therefore, the problem can be reduced to calculation of the dependence

$$
E=f(\eta) \text {. }
$$

If the long-range order parameter is smaller than unity, a part of the vacancies located in the vacancy sublattice of the $\mathrm{Ti}_{5} \mathrm{O}_{5}$ structure passes to the atomic sublattice, while a part of atoms from the atomic sublattice passes to the vacancy sublattice. Atoms and vacancies that have passed to the alien sublattice are distributed there randomly. The probability of finding an atom in the atomic sublattice is $5 / 6+\eta / 6$, while the probability of detecting an atom in the vacancy sublattice is $5 / 6-5 \eta / 6$ [23].

To simulate partly ordered structures, it is sufficient to use the supercell proposed in [17]. That supercell was obtained by double translation of the unit cell of the $\mathrm{Ti}_{5} \mathrm{O}_{5}$ ordered phase along crystallographic directions $a$ and $b$. It contains 96 sites of the $B 1$ basis structure, eight of which are occupied by titanium vacancies and another eight are occupied by oxygen vacancies. If $m$ atoms pass to the sublattice of vacancies, then long-range order parameter $\eta$ amounts to $6(5 / 6-m / 8) / 5$. Obviously, the fraction of atoms in the sublattice of vacancies cannot exceed their fraction in the completely disordered compound (i.e., 5/6). Then $m$ cannot be larger than 6 . Thus, transferring successively from 1 to 6 atoms from the atomic sublattice to the sublattice of vacancies, we can simulate the structure with different long-range order parameters (Table 2). In the case of the completely disordered structure $(\eta=0)$, the number of atoms in the sublattices of atoms and vacancies is not expressed as an integer; for this reason, this number was not fixed but

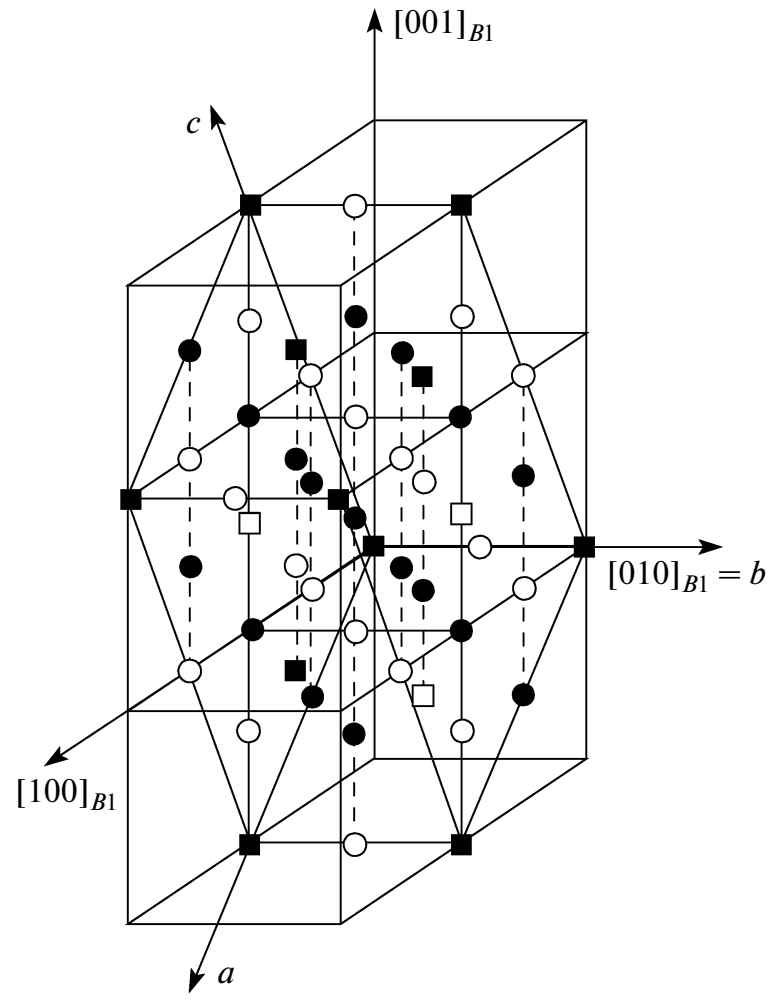

Fig. 1. Unit cell of the $\mathrm{Ti}_{5} \mathrm{O}_{5}$ superstructure [1,23]. Axes $a$, $b$, and $c$ of the superstructure and the direction of the $B 1$ basis structure are indicated. Black and white circles correspond to titanium and oxygen atoms, respectively, and black and open squares show vacancies in the titanium and oxygen sublattice, respectively.

was determined as a result of random distribution of atoms and vacancies over the sites of the sublattices in the basic structure. The concentration of atoms in the sublattices remained 5/6 like in the completely ordered phase. To take into account the random nature of the distribution of the transferred atoms and vacancies, we constructed ten supercells with different arrangements of the transferred atoms in the sublattice of vacancies and transferred vacancies in the atomic sublattice for each value of the long-range order parameter. The results of calculations for these supercells were averaged.

Table 1. Distribution functions for atoms and vacancies in the titanium and oxygen sublattices of the $\mathrm{Ti}_{5} \mathrm{O}_{5}$ monoclinic structure [23] $(x, z$ are the fracrtions of atomic positions in titanium and oxygen sublattices, respectively, of the $B 1$ basis structure)

\begin{tabular}{l|c}
\hline \multicolumn{1}{c|}{ Sublattice } & Distribution function \\
\hline Titanium & $n_{\mathrm{Ti}}=x+\eta^{\mathrm{Ti}} / 6$ \\
Titanium vacancies & $n_{\mathrm{Ti}}=x-5 \eta^{\mathrm{Ti}} / 6$ \\
Oxygen & $n_{\mathrm{O}}=z+\eta^{\mathrm{O}} / 6$ \\
Oxygen vacancies & $n_{\mathrm{O}}=z-5 \eta^{\mathrm{O}} / 6$ \\
\hline
\end{tabular}


Table 2. Number of atoms and vacancies in the supercell and corresponding values of long-range order parameter $\eta$ of the $\mathrm{Ti}_{5} \mathrm{O}_{5}$ superstructure

\begin{tabular}{c|c|c|c|c}
\hline $\begin{array}{c}\text { Number of atoms in } \\
\text { the titanium and } \\
\text { oxygen sublattices }\end{array}$ & $\begin{array}{c}\text { Number of atoms in } \\
\text { the sublattices of } \\
\text { titanium and oxygen } \\
\text { vacancies }\end{array}$ & $\begin{array}{c}\text { Number of vacancies } \\
\text { in the titanium and } \\
\text { oxygen sublattices }\end{array}$ & $\begin{array}{c}\text { Number of vacancies } \\
\text { in the sublattices of } \\
\text { titanium and oxygen } \\
\text { vacancies }\end{array}$ & $\begin{array}{c}\text { Value of long-range } \\
\text { order parameter } \eta\end{array}$ \\
\hline $140 / 3$ & $20 / 3$ & $20 / 3$ & $4 / 3$ & 0 \\
46 & 6 & 6 & 3 & $1 / 10$ \\
45 & 5 & 5 & 4 & $1 / 4$ \\
44 & 4 & 4 & 5 & $7 / 10$ \\
43 & 2 & 2 & 6 & $17 / 20$ \\
41 & 1 & 1 & 8 & 1 \\
\hline
\end{tabular}

The period of the basic lattice of the disordered phase was set at $418.2 \mathrm{pm}$, while the period of the ordered phase was assumed to be $414.2 \mathrm{pm}$ in accordance with the experimental data [21]. Period $a$ of the basic lattice for structures with intermediate values of the long-range order parameter was calculated from the equation of a straight line:

$$
a[\mathrm{pm}]=418.2-40 \eta .
$$

The total energy of the electron-nuclear system was calculated using density functional theory [25, 26] with the exchange-correlation potential of the generalized gradient approximation (GGA) correction (Perdew-Burke-Ernzerhof (PBE) version) [27]. The pseudopotential approach used in the calculations was developed on the basis of plane waves realized in the

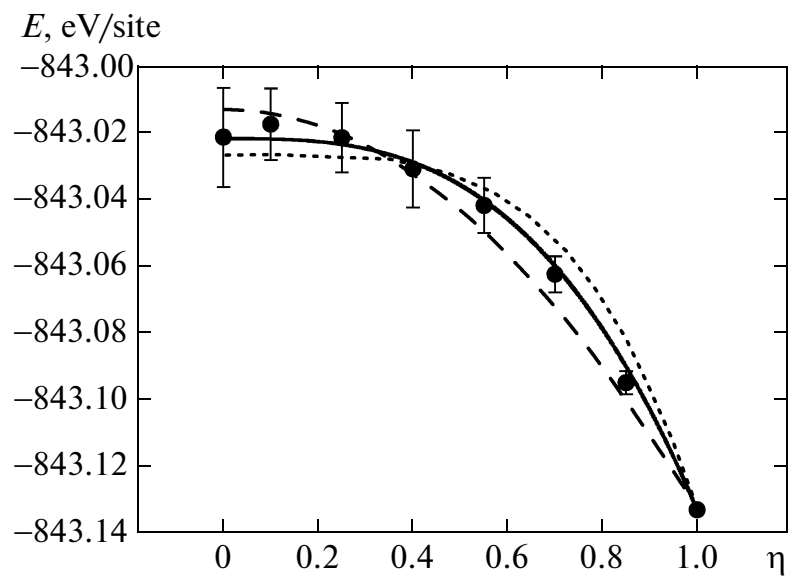

Fig. 2. Dependence of the internal energy of titanium monoxide on the long-range order parameter. The values of energy are calculated per site of the $B 1$ basis structure: - - results of calculations; dashed curve corresponds to $E=-843.013-0.120 \eta^{2}$; solid curve corresponds to $E=$ $-843.022-0.111 \eta^{3}$; dotted curve corresponds to $E=$ $-843.027-0.106 \eta^{4}$.
QUANTUM-ESPRESSO software package [28]. The kinetic energy of plane waves did not exceed $45 \mathrm{Ry}$. Apart from the valence $4 s 3 d$ states, the pseudopotential for titanium includes the $3 s 3 p$ core states. The appearance of vacancies in the structures leads to static displacements of atoms; for this reason, relaxation of atomic positions was carried out for each superlattice. The calculations were performed using the Uran supercomputer at the Institute of Mathematics and Mechanics, Ural Branch, Russian Academy of Sciences.

The results of calculating the internal energy of titanium monoxide per site of the $B 1$ basic lattice are shown in Fig. 2. The results of calculations are successfully described by the function

$$
E[\mathrm{eV}]=-843.022-0.111 \eta^{3}
$$

although the point charge model presumes the quadratic dependence [20]. Upon an increase of the longrange order parameter, the internal energy of the compound decreases. This means that at low temperatures, when the contribution of the entropy to the free energy is small, the ordered state of the system is energetically more advantageous as compared to the disordered state, which corresponds to experimental data [21-24].

The results of calculations make it possible to determine the enthalpies of formation $\Delta H$ by the formula

$$
\Delta H[\mathrm{~kJ} / \mathrm{mol}]=k\left(E-X E_{\mathrm{Ti}}-Y E_{\mathrm{O}_{2}} / 2\right) / 40,
$$

where $k$ is the conversion coefficient to $\mathrm{kJ} / \mathrm{mol} ; E$ is the total energy of titanium monoxide per supercell; $X$ and $Y$ are the numbers of titanium and oxygen atoms in the supercell, respectively; $E_{\mathrm{Ti}}$ is the total energy of metallic titanium per atom; $E_{\mathrm{O}_{2}}$ is the total energy of the oxygen molecule taking into account the spin polarization effect; and 40 is the number of structural units (Ti-O pairs) in the supercell. The enthalpy of formation of the completely disordered state amounts 


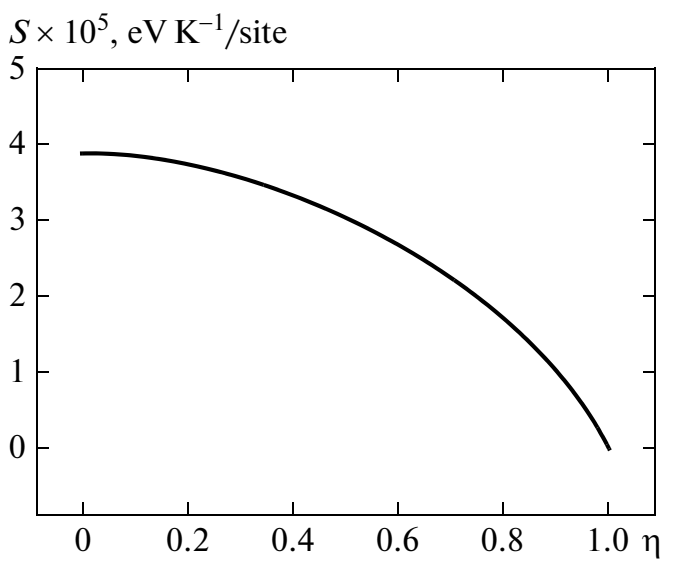

Fig. 3. Dependence of the configurational entropy of titanium monoxide on the long-range order parameter. The entropy values are calculated per site of the $B 1$ basis structure.

to $-521.8 \mathrm{~kJ} / \mathrm{mol}$ and agrees with the experimental value $(-518.4 \mathrm{~kJ} / \mathrm{mol})[18]$.

\section{CALCULATION OF ENTROPY}

The configurational entropy of the crystal, occurring as a result of disordered arrangement of atoms and vacancies, can be determined using the Boltzmann formula:

$$
S=k_{\mathrm{B}} \ln \Omega,
$$

where $\Omega$ is the number of microscopic states of the system by which the given macroscopic state is achieved. This value is determined for each of the four sublattices using the formula

$$
\Omega=\frac{M !}{K !(M-K) !},
$$

where $M$ is the number of sites in the sublattice under investigation and $K$ is the number of sites occupied by atoms in this sublattice.

The entropy of a crystal being ordered is the sum of the entropies of its sublattices. The entropies of the sublattices calculated using Stirling's formula as functions of the long-range order parameters are given in [20]. Taking into account the condition $\eta^{\mathrm{Ti}}=\eta^{\mathrm{O}}=\eta$, the configurational entropy per site of the $B 1$ basic structure is expressed as

$$
\begin{aligned}
S[\mathrm{eV} / \mathrm{K}] & =8.617 \times 10^{-5}\left[\frac { 5 } { 6 } \left(-\left[\frac{5}{6}+\frac{\eta}{6}\right] \ln \left[\frac{5}{6}+\frac{\eta}{6}\right]\right.\right. \\
-(1 & \left.\left.-\left[\frac{5}{6}+\frac{\eta}{6}\right]\right) \ln \left(1-\left[\frac{5}{6}+\frac{\eta}{6}\right]\right)\right) \\
+ & \frac{1}{6}\left(-\left[\frac{5}{6}-\frac{5 \eta}{6}\right] \ln \left[\frac{5}{6}-\frac{5 \eta}{6}\right]\right.
\end{aligned}
$$

$F, \mathrm{eV} /$ site

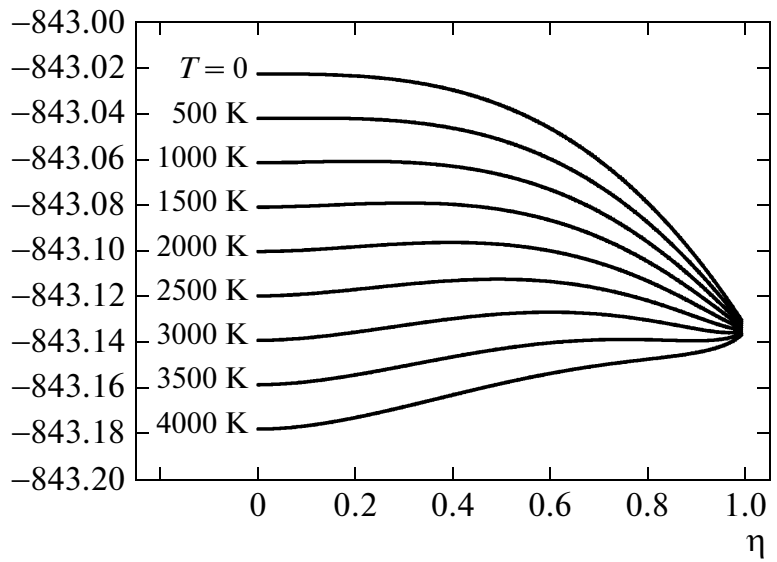

Fig. 4. Dependence of the free energy of titanium monoxide on the long-range order parameter. The values of entropy are calculated per site of the $B 1$ basis structure.

$$
\left.\left.-\left(1-\left[\frac{5}{6}-\frac{5 \eta}{6}\right]\right) \ln \left(1-\left[\frac{5}{6}-\frac{5 \eta}{6}\right]\right)\right)\right] .
$$

This dependence is shown graphically in Fig. 3.

\section{FREE ENERGY}

Figure 4 shows the dependences of the free energy of titanium monoxide being ordered on the long-range order parameter, calculated using expressions (3), (9), and (14) for various temperatures. The shape of the curves considerably depends on temperature. The minimal value of the internal energy is attained at point $\eta=1$ at low temperatures and at the extremum points or at $\eta=0$ at high temperatures. It should be noted that in contrast to the results obtained in accordance with the point charge model, the points of the extremum of the free energy functions are considerably shifted towards the highest long-range order parameters (Fig. 5).

In the single-phase domain, the structure with a long-range order parameter corresponding to the minimal possible free energy at the given temperature is in thermodynamic equilibrium. To determine these equilibrium values of the long-range order parameters, it is necessary to compare the values of the free energy functions at the points of extremum with the values at points $\eta=1$ and $\eta=0$. The search for the positions of the extrema was carried out using the computer method for scanning functions. The temperature dependence of the equilibrium values of long-range order parameter is shown in Fig. 6 .

Calculations have shown that the ordered state of titanium monoxide must be in thermodynamic equilibrium in the temperature interval from 0 to $2903 \mathrm{~K}$. At higher temperatures, only the disordered state with $\eta=0$ is in equilibrium. The order-disorder phase transition must occur in accordance with the mecha- 


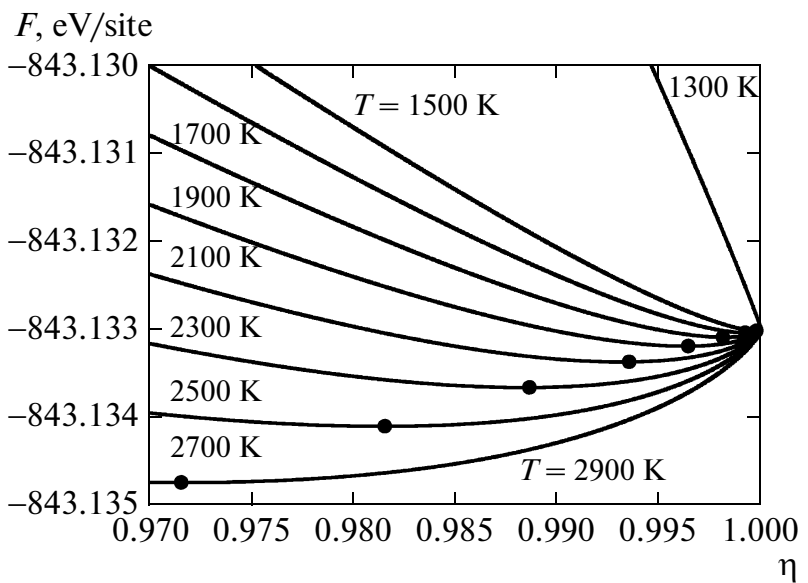

Fig. 5. Temperature dependence of equilibrium values of the long-range order parameter. At temperatures above the order-disorder phase transition temperature, the equilibrium values of the long-range order parameters are zero.

nism of the first-order phase transition with a critical value of the long-range order parameter $\eta=0.971$. At the same time, the point charge model gives a critical value of the long-range order parameter of 0.6 and a phase-transition temperature an order of magnitude higher than that calculated in this study [20]. On the other hand, the phase transition temperature of $2903 \mathrm{~K}$ is more than twice as high as the experimental value. Since the pseudopotential approach used here gives adequate energy parameters for the compound, which is confirmed by agreement with experiment [18], a possible reason for the discrepancy between the calculated and actual phase-transition temperature lies in the error in the entropy estimate. In this study, like in other analogous cases [1-6, 10-16], we take into account only the contribution of the configurational entropy of the compound being ordered. However, the result shows that its contribution (at least for titanium monoxide) is obviously insufficient to decrease the calculated temperature to $1263 \mathrm{~K}$. Therefore, along with configurational entropy, the dependence of the phonon entropy on the long-range order parameters must be taken into account in calculating the equilibrium phase diagrams of strongly nonstoichiometric compounds. At present, direct calculation of the phonon entropy for supercells of the size used in this study is impossible because of insurmountable computational difficulties. This necessitates the development of convenient approaches and approximations for taking into account the phonon entropy in thermodynamic calculations.

Another reason may be the incorrect notion about the actual structure of the compound under investigation. In the model considered here, atoms were placed during disordering at randomly selected sites of the basal structure. As a matter of fact, correlations between the atoms must lead to the occurrence of short-range order, which may change the contribu-

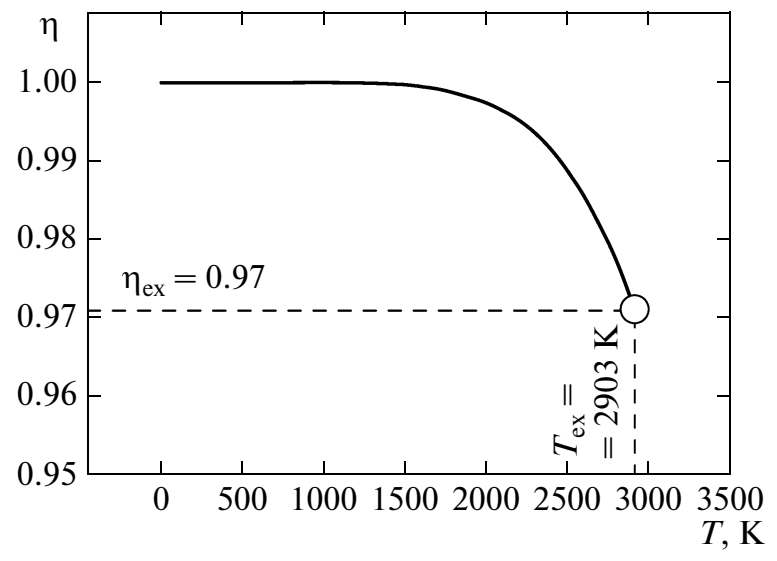

Fig. 6. Positions of extrema of the free energy functions at various temperatures.

tions of the internal energy as well as of the configurational entropy to the free energy of the compound. The supercell method and the cluster approach make it possible to take these contributions into account, but this requires exact information on correlations in the positions of vacancies at least in the first three coordination spheres. However, no concrete models have been proposed for the structure of nonstoichiometric compounds with a short-range order in the arrangement of vacancies as yet; therefore, the shortrange order could not be taken into account in this study.

\section{CONCLUSIONS}

Thus, we have analyzed the dependence of the free energy on the long-range order parameter for $\mathrm{TiO}_{y}$, which represents a class of strongly nonstoichiometric compounds. This dependence has been determined on the basis of energy parameters of the lattice, obtained from quantum-mechanical ab initio calculations. Analysis of the free energy of titanium monoxide $\mathrm{TiO}_{y}$ of equiatomic composition has shown that the orderdisorder phase transition must occur in accordance with the mechanism of a first-order phase transition at a temperature of $2903 \mathrm{~K}$. The critical value of the longrange order parameter is close to unity and amounts to 0.971 . The calculated phase-transition temperature is an order of magnitude lower than the temperature predicted by the point charge model, but it is more than twice as high as the experimental value.

\section{ACKNOWLEDGMENTS}

The authors are grateful to A.A. Valeeva for her interest in this research and valuable remarks.

This study was financially supported by Ural Branch of the Russian Academy of Sciences (project nos. 12-M-23-2001 (together with the Institute of 
Metal Physics, Ural Branch, Russian Academy of Sciences) and 13-3-NP-532 (young scientists)).

\section{REFERENCES}

1. A. I. Gusev and A. A. Rempel', Nonstoichiometry, Disorder, and Order in Solids (Ural Branch of the Russian Academy of Sciences, Yekaterinburg, 2001) [in Russian].

2. R. Kikuchi, Phys. Rev. 81, 988 (1951).

3. M. Kurata, R. Kikuchi, and T. Watari, J. Chem. Phys. 21, 434 (1953).

4. J. Hijmans and J. de Boer, Physica (Amsterdam) 21, 471 (1955).

5. R. Kikuchi and S. G. Brush, J. Chem. Phys. 47, 195 (1967).

6. A. A. Rempel', Effects of Ordering in Nonstoichiometric Interstitial Compounds (Nauka, Yekaterinburg, 1992).

7. A. A. Valeeva and A. A. Rempel', JETP Lett. 88 (3), 172 (2008).

8. M. G. Kostenko, A. A. Valeeva, and A. A. Rempel, Mendeleev Commun. 22, 245 (2012).

9. M. G. Kostenko and A. A. Rempel, JETP 115 (6), 999 (2012).

10. A. I. Gusev and A. A. Rempel, Phys. Status Solidi B 131, 43 (1985).

11. A. I. Gusev and A. A. Rempel', Zh. Fiz. Khim. 60, 1349 (1986).

12. A. I. Gusev and A. A. Rempel, Phys. Status Solidi B 140, 335 (1987).

13. A. I. Gusev and A. A. Rempel', Zh. Fiz. Khim. 65, 625 (1991).

14. A. I. Gusev and A. A. Rempel, J. Phys. Chem. Solids 55, 299 (1994).

15. A. I. Gusev and A. A. Rempel, Phys. Status Solidi A 163, 273 (1997).
16. A. I. Gusev and A. A. Rempel, in Materials Science of Carbides, Nitrides, and Borides, Ed. by Y. G. Gogotsi and R. A. Andrievski (Kluwer, Dordrecht, 1999), p. 47.

17. M. G. Kostenko, A. V. Lukoyanov, V. P. Zhukov, and A. A. Rempel', JETP Lett. 95 (12), 647 (2012).

18. G. L. Humprey, J. Am. Chem. Soc. 73, 1587 (1951).

19. D. A. Andersson, P. A. Korzhavyi, and B. Johansson, Phys. Rev. B: Condens. Matter 71, 144101-1 (2005).

20. M. G. Kostenko and A. A. Rempel', Phys. Solid State 53 (9), 1909 (2011).

21. D. Watanabe, J. R. Castles, A. Jostsons, and A. S. Marlin, Acta Crystallogr. 23, 307 (1967).

22. D. Watanabe, O. Terasaki, A. Jostsons, and J. R. Castles, in The Chemistry of Extended Defects in NonMetallic Solids, Ed. by L. Eyring and M. O'Keefe (North-Holland, Amsterdam, 1970), p. 238.

23. A. A. Valeeva, A. A. Rempel', and A. I. Gusev, Inorg. Mater. 37 (6), 603 (2001).

24. D. Watanabe, O. Terasaki, A. Jostsons, and J. R. Castles, J. Phys. Soc. Jpn. 25, 292 (1968).

25. W. Kohn and L. J. Sham, Phys. Rev. [Sect.] A 140, 1133 (1965).

26. R. O. Jones and O. Gunnarsson, Rev. Mod. Phys. 61, 689 (1989).

27. J. P. Perdew, K. Burke, and M. Ernzerhof, Phys. Rev. Lett. 77, 3865 (1996).

28. P. Giannozzi, S. Baroni, N. Bonini, M. Calandra, R. Car, C. Cavazzoni, D. Ceresoli, G. L. Chiarotti, M. Cococcioni, I. Dabo, A. D. Corso, S. de Gironcoli, S. Fabris, G. Fratesi, R. Gebauer, U. Gerstmann, C. Gougoussis, A. Kokalj, M. Lazzeri, L. MartinSamos, N. Marzari, F. Mauri, R. Mazzarello, S. Paolini, A. Pasquarello, L. Paulatto, C. Sbraccia, S. Scandolo, G. Sclauzero, A. P Seitsonen, A. Smogunov, P. Umari, and R. M. Wentzcovitch, J. Phys. Condens. Matter 21, 395502 (2009).

Translated by N.V. Wadhwa 